\title{
Ultra-High Energy Neutrino-Nucleon Scattering
}

\author{
Ernest M. Henley and W-Y. P. Hwang ${ }^{\mathrm{b}}$ \\ ${ }^{a}$ Department of Physics, and Institute for Nuclear Theory, \\ University of Washington, Seattle, WA 98195, USA
}

${ }^{\mathrm{b}}$ Department of Physics, National Taiwan University, Taipei, Taiwan RC

For ultra high energy neutrinos, does the deep inelastic cross section for the scattering from nucleons grow so fast that the unitarity limit is reached?

Deep inelastic cross sections from nucleons at ultra-high energies are dominated by the gluons in the nucleon. It is known from HERA data for electron scattering that the structure functions, e.g., $F_{2}$, increases as the momentum fraction $x$ decreases. The gluon density gets to be very high at small Feynman $x$. It is then to be expected that the partons interact with each other and this effect will lead to Glauber-type shadowing and to saturation of $F_{2}$ and of the neutrino-nucleon cross section. The saturation scale has been parameterized by Golec-Biernat and Wüsthoff[1]. A semiphenomenological analysis has shown that the $q \bar{q}$ color dipole formalism can be used and explains saturation at very small $x$. We use the color glass condensate and the quenched or screened version of Bartels et al.[1]. DGLAP evolution is used. The differential neutrino-nucleon cross section can be written in terms of the structure functions $F_{1}$ and $F_{2}$ [2] ( $F_{3}$ does not contribute at small $\mathrm{x}$ ).

Our results for $F_{2}$ at $Q^{2}=M_{W}^{2}$ can be summarized as follows: It rises from 10 at $x=10^{-3}$ to 36 at $x=10^{-5}$ and to 145 at $x=10^{-7}$. By contrast, that obtained by Kutak and Kwiecińsky[3] rises more sharply for small $\mathrm{x}$ for both their screened and unscreened BFKL/DGLAP evolution. Our total cross section for deep inelastic scattering with two contributing flavors $(d \leftrightarrow u, s \leftrightarrow c)$ is given in the Table below.

\begin{tabular}{|c|c|}
\hline Energy (GeV) & Cross Section $\left(\mathrm{cm}^{2}\right)$ \\
\hline $10^{6}$ & $1.2 \times 10^{-33}$ \\
$10^{8}$ & $7.9 \times 10^{-33}$ \\
$10^{10}$ & $3.3 \times 10^{-32}$ \\
$10^{12}$ & $1.2 \times 10^{-31}$ \\
\hline
\end{tabular}

Our result is approximately twice that of ref.[3] with screening and thus somewhat larger than theirs without screening. Our cross section is somewhat larger than that of Gandhi et al.[4] at $10^{6} \mathrm{GeV}$, but grows more slowly with energy and agrees with theirs at $10^{12} \mathrm{GeV}$. They do not consider saturation.

The authors thank Jamal Jalilian-Marian for numerous discussions and Mary Alberg and William Detmold for some computing help. This work has been supported, in part, by the Department of Energy (U.S.) and by the Ministry of Education (Taiwan).

\section{REFERENCES}

1. See e.g., K. Golec-Biernat and M. Wüsthoff, Phys. Rev D60 (1999), 114023; J. Bartels, K. Golec-Biernat, and H. Kowalski, Phys. Rev. D66 (2002), 014001

2. J. Jalilian-Marian , Phys. Rev 68 (2003), 054005

3. K. Kutak and J. Kwieciński, Eur. Phys. J. C29 (2003), 521

4. R. Gandhi , C. Quigg, M.H. Reno, and I Sarcevic, Phys. Rev D58 (1998), 093009. 